\title{
Differential changes of metabolic brain activity and interregional functional coupling in prefronto-limbic pathways during different stress conditions: functional imaging in freely behaving rodent pups
}

\author{
Jörg Bock ${ }^{1,2}$, Anett Riedel ${ }^{3}$ and Katharina Braun ${ }^{1,3}$ \\ ${ }^{1}$ Center for Behavioral Brain Sciences Magdeburg, Magdeburg, Germany \\ 2 PG Structural Plasticity, Institute of Biology, Otto-von-Guericke University, Magdeburg, Germany \\ ${ }^{3}$ Department of Zoology/Developmental Neurobiology, Institute of Biology, Otto-von-Guericke University, Magdeburg, Germany
}

Edited by:

Nicola Maggio, The Chaim Sheba

Medical Center, Israel

\section{Reviewed by:}

Mathias V. Schmidt, Max Planck Institute of Psychiatry, Germany Aron Weller, Bar-Ilan University, Israel

\section{${ }^{*}$ Correspondence:}

Jörg Bock, PG Structural Plasticity,

Institute of Biology, Otto-von-

Guericke University, Leipziger

Str. 44, Magdeburg, Germany.

e-mail: joerg.bock@ovgu.de
The trumpet-tailed rat or degu (Octodon degus) is an established model to investigate the consequences of early stress on the development of emotional brain circuits and behavior. The aim of this study was to identify brain circuits, that respond to different stress conditions and to test if acute stress alters functional coupling of brain activity among prefrontal and limbic regions. Using functional imaging (2-Fluoro-deoxyglucose method) in 8-day-old male degu pups the following stress conditions were compared: (A) pups together with parents and siblings (control), (B) separation of the litter from the parents, (C) individual separation from parents and siblings, and (D) individual separation and presentation of maternal calls. Condition (B) significantly downregulated brain activity in the prefrontal cortex, hippocampus, nucleus accumbens (NAcc), and sensory areas compared to controls. Activity decrease was even more pronounced during condition (C), where, in contrast to all other regions, activity in the PAG was increased. Interestingly, brain activity in stress-associated brain regions such as the amygdala and habenula was not affected. In condition (D) maternal vocalizations "reactivated" brain activity in the cingulate and precentral medial cortex, NAcc, and striatum and in sensory areas. In contrast, reduced activity was measured in the prelimbic and infralimbic cortex (IL) and in the hippocampus and amygdala. Correlation analysis revealed complex, region- and situation-specific changes of interregional functional coupling among prefrontal and limbic brain regions during stress exposure. We show here for the first time that early life stress results in a widespread reduction of brain activity in the infant brain and changes interregional functional coupling. Moreover, maternal vocalizations can partly buffer stress-induced decrease in brain activity in some regions and evoked very different functional coupling patterns compared to the three other conditions.

Keywords: limbic system, prefrontal cortex, functional imaging, functional coupling, stress, PAG, maternal separation

\section{INTRODUCTION}

The critical role of early adverse life experience, in addition to a genetic contribution, has been postulated to contribute to the susceptibility and pathogenesis of mood and anxiety disorders. There is clear evidence that early life stress, such as the separation of the newborn from its mother or parents as well as physical, sexual, emotional or verbal abuse all affect the maturation of endocrine and behavioral function in rodents, non-human primates, and humans (Korosi and Baram, 2009; Heim et al., 2010; Neumann et al., 2010; Pechtel and Pizzagalli, 2010; Bock and Braun, 2011; Pryce et al., 2011; Schmidt et al., 2011). The majority of studies on the neurobiological effects of stress exposure are focused on chronic stress in adulthood and its endocrine effects, its impact on the adult brain was mainly analyzed in the hippocampal formation (Sandi, 2004; Fuchs et al., 2006; Guterman and Richter-Levin, 2006; McEwen, 2010). Much less is known about the impact of acute, or repeated stress exposure on the immature developing brain, and on the functional maturation of prefrontal and limbic circuitries, which mediate perceptive, interpretative, and controlling aspects of emotionality. Due to the high vulnerability of the immature brain and the associated risks to develop dysfunctions we need to gain a more detailed understanding of the immediate and long-term neurobiological effects of early life stress, for example, child abuse and neglect or parental loss, in particular its long-term impact on the immature brain.

The trumpet-tailed rat Octodon degus is an established animal model to study the development of social behavior and emotional experience during postnatal and adolescent development (Colonnello et al., 2011) and to analyze the impact of early life stress on the development of prefronto-limbic brain circuits (Bock and Braun, 2011; Braun and Bock, 2011). This precocious, 
diurnal South American rodent lives in complex social family structures, families are biparental and degu pups have been shown to develop a strong attachment to both parents (Fuchs et al., 2010). Similar to humans degus use an elaborated vocal communication system among family and colony members (Braun and Scheich, 1997).

So far, only little is known about the brain circuits and their networks, which are involved in information processing during or after exposure to positive or adverse emotional situations in the infant brain. One recent study, using cytochrome oxidase activity as a measure of long-term changes in brain metabolic capacity after 2 weeks of exposure to repeated separation stress, reports decreased activity in the medial prefrontal cortex and nucleus accumbens (NAcc) in 2-week-old mouse pups (Spivey et al., 2011). A PET study in young rhesus monkeys showed that acute maternal separation is associated with an activation in the right dorsolateral prefrontal cortex and ventral temporal/occipital lobes and decreased activity in the left dorsolateral prefrontal cortex (Rilling et al., 2001).

One aim of this functional imaging study was to systematically map metabolic activations/deactivations in the infant degu brain during different degrees of acute separation stress and thereby identify the underlying brain circuits in the infant brain. Since functionally correlated neuronal activity is essential for the activity-dependent maturation of neuronal networks, and deviations of these networks may contribute to the etiology of neurodevelopmental disorders (Uhlhaas et al., 2009; Stam and van Straaten, 2012), we also aimed to identify interregional functional coupling among the stress-responsive brain circuitries. In view of the recently emerging literature from human imaging studies exploring functional network connectivity to assess their involvement in different emotional states, the paucity of network analyses in animal models related to stress is surprising. We hypothesize that separation stress induces widespread alterations of metabolic brain activity and alters the interregional functional coupling among prefrontal and limbic brain regions. We also predict that the magnitude of the stress-induced metabolic changes should correlate to the degree of stress. Another aim was to test the hypothesis that a positive emotional stimulus, the maternal voice, which on the behavioral level has been shown to exert an "anxiolytic" effect (Braun et al., 2003; Ziabreva et al., 2003a,b) should ameliorate, restore or "normalize" stressinduced metabolic changes. Since functional MRI per se is a stressful procedure, and therefore requires sedation in the animals, we applied the 2-Fluoro-deoxy-glucose (FDG)-technique, which allows to quantify changes of regional metabolic activity in awake, freely behaving animals (Gonzalez-Lima and Scheich, 1986; Wallhäusser and Scheich, 1987; Gonzalez-Lima, 1992; Bock et al., 1996, 1997; Nair et al., 2001; Moriceau and Sullivan, 2006; Riedel et al., 2010).

\section{MATERIALS AND METHODS ANIMALS}

The degus were bred in our colony at the Leibniz Institute for Neurobiology (Magdeburg, Germany). Family groups of an adult couple and their offspring were housed in large wire cages $(100 \mathrm{~cm} \times 84 \mathrm{~cm} \times 40 \mathrm{~cm})$ and exposed to a $12 \mathrm{~h} \mathrm{light} / 12 \mathrm{~h}$ dark cycle. Food and drinking water were available ad libitum. The rooms were maintained at an average temperature of $22^{\circ} \mathrm{C}$. After the birth of the pups the home cages were not cleaned until the start of the experiments to avoid unspecific exposure to stressors (disturbing the cage) and handling.

All experiments were performed in accordance with the European Communities Council Directive of November 24, 1986 (86/609/EEC) and according to the German guidelines for the care and use of animals in laboratory research. The experimental protocols were approved by the ethics committee of the government of the state of Saxony-Anhalt.

\section{EXPERIMENTAL GROUPS}

All pups were reared in their natural family groups, i.e., together with their parents and siblings until the start of the experiments. All experiments were conducted between 11 am and 2 pm during the light phase (degus are diurnal, i.e., active during the day phase). For 2-FDG experiments an overall of 16 male degu pups from four families (litter size 7-8 animals) were analyzed at postnatal day (PND) 8. Due to the limited availability of degus (each breeding couple produces only 2 litters per year due to three months gestational period, and an extended weaning period) this experimental design does not completely exclude litter effects.

The pups were divided into four experimental groups of four siblings as follows:

"parents" (control): Pups of this unstressed control group were left undisturbed together with their parents and siblings in the home cage.

"litter separation": Pups of this group were exposed to separation stress by removing them from their home cage and their parents as a group of littermates, which were transferred to a paperboard box $(25 \times 25 \mathrm{~cm})$, where they were left undisturbed for the duration of the 2-FDG experiment $(1 \mathrm{~h})$.

"individual separation": Pups of this group were exposed to separation stress as described for "litter separation", however, the pups of this group were also separated from their siblings and kept individually in paperboard boxes, where they were left undisturbed for the duration of the 2-FDG experiment $(1 \mathrm{~h})$. During the experiment no visual or tactile, but acoustic and olfactory contact among the siblings was possible.

"individual separation + call": Pups of this group were separated as described for "individual separation," but to ameliorate their stress levels maternal vocalizations were presented from a loudspeaker during the separation period (Braun and Poeggel, 2001; Ziabreva et al., 2003a,b). The presented vocalizations were "mothering calls", which the dams exclusively use when they have pups and which serves to attract the pups and stimulate their suckling behavior. The acoustic features of the maternal attraction calls and the metabolic activation patterns in the auditory cortex (AC) of degu pups were described in detail in Braun and Scheich (1997). It is important to note that all pups were stimulated with the same maternal calls, i.e., the calls were not from their own mother. The auditory stimulation was as follows: $10 \mathrm{~s}$ stimulation, $20 \mathrm{~s}$ break. This stimulation protocol was repeated during the entire imaging experiment. 


\section{2-FLUORODEOXYGLUCOSE AUTORADIOGRAPHY}

The stimulus-evoked brain activation patterns were analyzed applying the 2-deoxyglucose method developed by Sokoloff et al. (1977). Due to its better penetration through the blood-brain barrier and the higher rate of phosphorylation we used 2-fluoro2-deoxy-D-[U-14C]glucose (2-FDG) (Amersham) as originally described by Gonzalez-Lima (Gonzalez-Lima, 1992) in our work (Bock et al., 1996, 1997; Poeggel and Braun, 1997; Riedel et al., 2010). Pups of either experimental group were weight and immediately thereafter received intraperitoneal injections of 2-FDG, $6 \mu \mathrm{Ci}$ /animal in $0.2 \mathrm{ml}$ sterile saline and were then exposed to the respective experimental condition for $1 \mathrm{~h}$. After the experiment degu pups were decapitated, the brains removed from the skull and rapidly frozen on the freezing mount of a cryostat. Serial transverse $40 \mu \mathrm{m}$ cryosections were collected on microscope slides, rapidly dried on a heating plate at $50^{\circ} \mathrm{C}$, pressed on Kodak NMB1 X-ray film in Kodak-X-omatic cassettes, and exposed for 2 weeks.

2-FDG-uptake was analyzed using an image analysis system consisting of a computer equipped with the public domain software NIH Image/ImageJ and a high resolution CCD-camera (Grundig FA 85 I, Fürth, Germany). The following brain areas were analyzed (according to Paxinos and Watson, 1998):

1. Frontal cortical areas: anterior cingulate cortex (ACd, according to Cg1 rostral to the corpus callosum), prelimbic cortex (PL), infralimbic (IL), orbitofrontal cortex (OFC, consisting of the ventral and lateral OFC), posterior cingulate cortex $(\mathrm{Cg}$, according to $\mathrm{Cg} 1$ and $\mathrm{Cg} 2$ dorsal to the corpus callosum), precentral medial cortex (PrCm, according to M2 rostral to the corpus callosum).

2. Limbic brain areas: dorsal hippocampus (hippo), lateral amygdala (LA), basomedial amygdala (BMA), Nucleus accumbens (NAcc).

3. Sensory and subcortical areas: dorsal striatum, somatosensory cortex (SSC), auditory cortex (AC), dorsal thalamus (thalamus), habenula, periaqueductal gray (PAG).

For each brain area five sections were measured and the values were averaged for statistical analysis. Since no significant differences of 2-FDG-incorporation between the left and right hemisphere were detectable the measurements of both hemispheres were pooled.

In order to compensate for metabolic differences between individual animals 2-FDG-incorporation was expressed as density ratio relative to the uptake in the corpus callosum (relative optical density, rOD). The corpus callosum was selected as reference area, because as a region of white matter it generally displays a uniform, stimulus-independent low glucose utilization. Optical density of the corpus callosum did not vary significantly across the experimental groups.

\section{STATISTICAL ANALYSIS}

Data analysis and diagram compilation were performed with SigmaPlot 11 (Systat Software GmbH, Erkrath, Germany) and JMP, Release 7 (SAS Institute Inc.; Cary/NC, USA). Differences in the density ratios across the experimental groups were analyzed by using a Kruskal-Wallis One-Way ANOVA (degrees of freedom = 3), which was followed by a Mann-Whitney $U$-test for pair-wise comparisons.

In addition to the group comparisons, patterns of "correlated functional activity" were calculated for every single behavioral condition. This was achieved by calculating the correlation coefficients between the rODs (inter-regional correlations). The correlations were performed using Spearman's rank correlation coefficient, usually called "Spearman's rho $(\rho)$," which is a measure of statistical dependencies between non-parametric variables. A Spearman correlation of 1 results when the two variables are monotonically-not linearly-related ( -1 indicates significant inverse relationship). To illustrate the strengths and direction of the inter-regional correlations, mosaic plots were compiled $(\alpha=5 \%)$.

\section{RESULTS}

\section{GROUP COMPARISONS OF METABOLIC BRAIN ACTIVITY}

One-Way ANOVA revealed significant differences in the metabolic brain activity between the experimental animals in the frontal cortical areas ACd $(p \leq 0.001, H=20.701)$, $\mathrm{Cg}(p \leq 0.001, H=25.639)$, IL $(p \leq 0.001, H=22.037), \mathrm{PL}$ $(p \leq 0.001, H=25.605) \operatorname{PrCm}(p \leq 0.001, H=23.006)$, and OFC $(p \leq 0.05, H=7853)$, the limbic areas hippocampus $(p \leq 0.001, H=22.748)$, LA $(p=0.032, H=8.803)$, and BMA ( $p \leq 0.002, H=14.707)$ and the sensory and subcortical areas dorsal striatum $(p \leq 0.001, H=24.692)$, NAcc $(p \leq 0.001$, $H=20.306)$, SSC $(p \leq 0.001, H=21.105)$, AC $(p \leq 0.001$, $H=17.344)$, dorsal thalamus $(p \leq 0.001, H=23.009)$ and $\mathrm{PAG}$ $(p \leq 0.002, H=14.888)$. No significant alterations were found in the habenula.

Applying a Mann-Whitney $U$-test for post-hoc pair-wise statistical analysis we observed the following differences between the individual experimental groups:

\section{EFFECTS OF SEPARATION STRESS ON METABOLIC BRAIN ACTIVITY Frontal cortical areas}

In general, separation stress decreased metabolic activity in all analyzed prefrontal cortical areas. Compared to unstressed controls ("parents") a significantly $(p \leq 0.05)$ reduced brain activity was measured in the ACd, Cg, PL, and PrCm of the "litter separation" and "individual separation" group (Figures 1 and 4). In the IL only the "individual separation" group showed a significant decrease compared to the "parents" group. No significant differences were found in the OFC. Compared to the pups from the "litter separation" group the "individual separation" group showed a more pronounced ( $p \leq 0.05$ ) decrease of brain activity in the Cg, PL and PrCm but not in the ACd (Figures 1 and 4).

\section{Limbic brain areas}

Similar to the observations in the frontal cortical areas a significant $(p \leq 0.05)$ reduction of brain activity was measured in the hippocampus and NAcc of the "litter separation" and "individual separation" group compared to the unstressed "parents" group (Figures 2 and 4). Again, the "individual separation" group showed a more pronounced ( $p=0.05$ ) reduction of brain activity in the hippocampus and NAcc compared to the "litter separation" group. No significant stress-induced differences were found in the amygdala (LA and BMA) and in the habenula (Figure 2). 

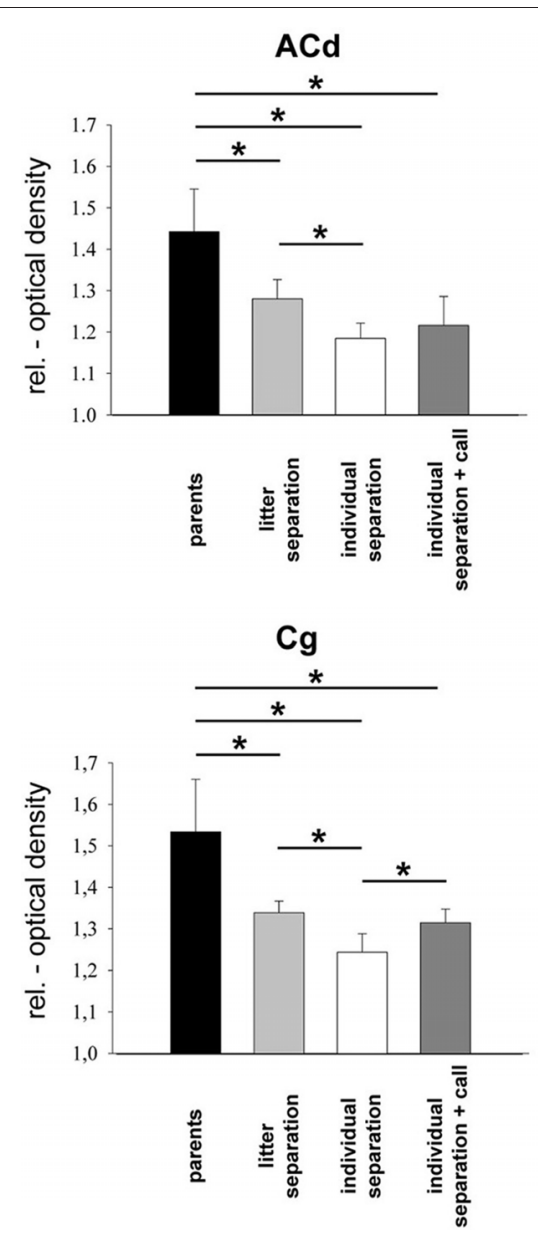
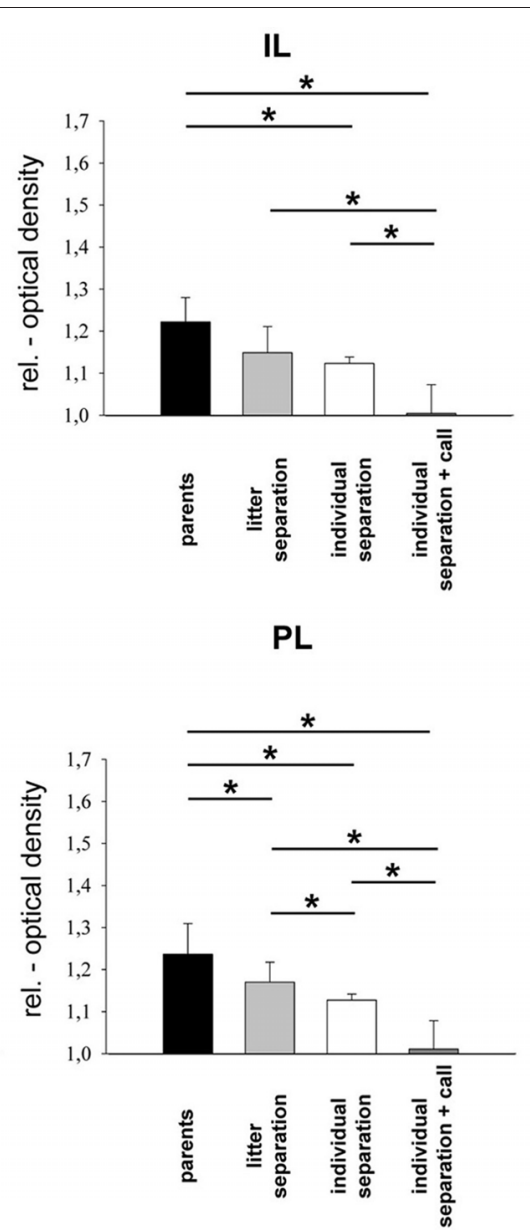
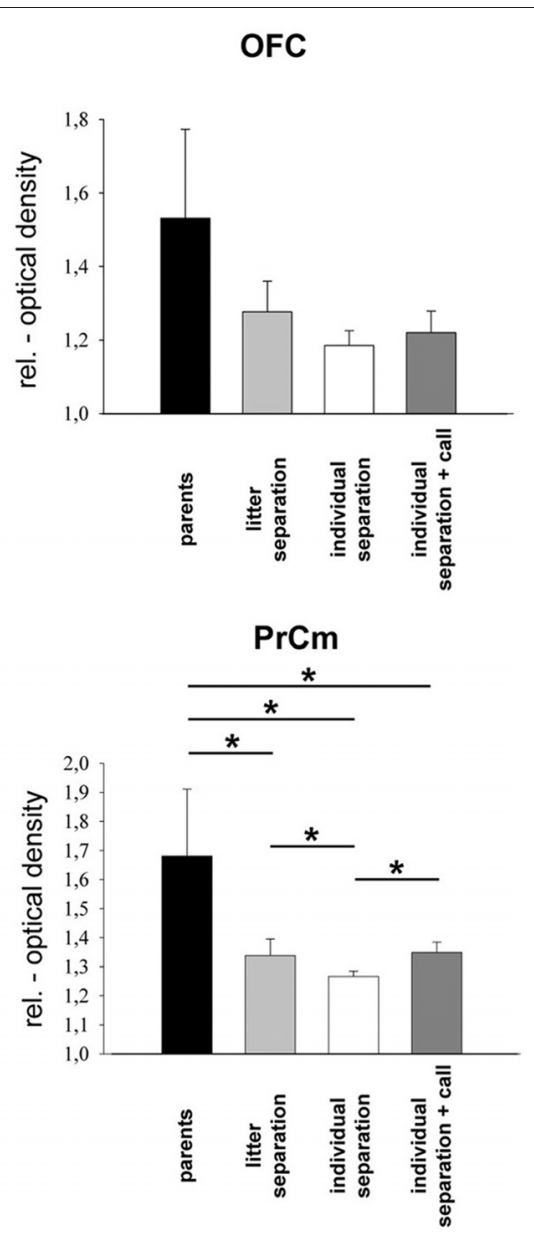

FIGURE 1 | Relative optical density of frontal cortical areas in the four experimental groups. * $p \leq 0.05$, Mann-Whitney $U$-test. ACd, anterior cingulate cortex; Cg, cingulate cortex; IL, infralimbic cortex; OFC, orbitofrontal cortex; PL, prelimbic cortex; PrCm, precentral medial cortex.

\section{Sensory and subcortical areas}

In the dorsal striatum, somatosensory and AC and in the dorsal thalamus a significant $(p \leq 0.05)$ reduction of brain activity was measured in the "litter separation" and "individual separation" group compared to the unstressed "parents" group (Figures 3 and 4). Also, in the dorsal striatum, SSC and thalamus the "individual separation" group showed a more pronounced reduction of brain activity compared to the "litter separation" group ( $p \leq$ $0.05)$. In contrast to all other brain areas the PAG showed a significant increase of brain activity in the "individual separation" group compared to the "parents" and "litter separation" group $(p \leq 0.05)$ (Figure 3).

\section{MATERNAL VOCALIZATIONS ALTER STRESS-INDUCED CHANGES IN METABOLIC BRAIN ACTIVITY}

Frontal cortical areas

In the $\mathrm{PrCm}$ and $\mathrm{Cg}$ the presentation of maternal vocalizations during exposure to separation stress ("individual separation + call" group) resulted in a significant $(p \leq 0.05)$ increase of brain activity compared to the "individual separation" group (Figure 1). In line with our prediction metabolic activity in the
PrCm and Cg was upregulated in the "individual separation + call" group to similar levels as observed in the "litter separation group." The ACd of the "individual separation + call" group displayed reduced brain activity compared to the unstressed "parents" group $(p \leq 0.05)$.

Opposite effects were observed in the IL and PL, where the presentation of maternal vocalizations induced a further downregulation of brain activity compared to the three other experimental groups $(p \leq 0.05)$ (Figure 1). Again, no significant effects were observed in the OFC.

\section{Limbic brain areas}

Similar to the findings for the prefrontal PL and IL presentation of maternal vocalizations during individual separation resulted in a significant ( $p \leq 0.05$ ) decrease of brain activity in the hippocampus and in the BMA compared to the three other experimental groups (Figure 2). In the LA a significant decrease of brain activity was observed only compared to the "litter separation" and "parents group." In contrast, the NAcc of the "individual separation + call" group showed a significant $(p=0.05)$ increase of brain activity compared to the "individual separation" group. No significant effects were observed in the habenula. 

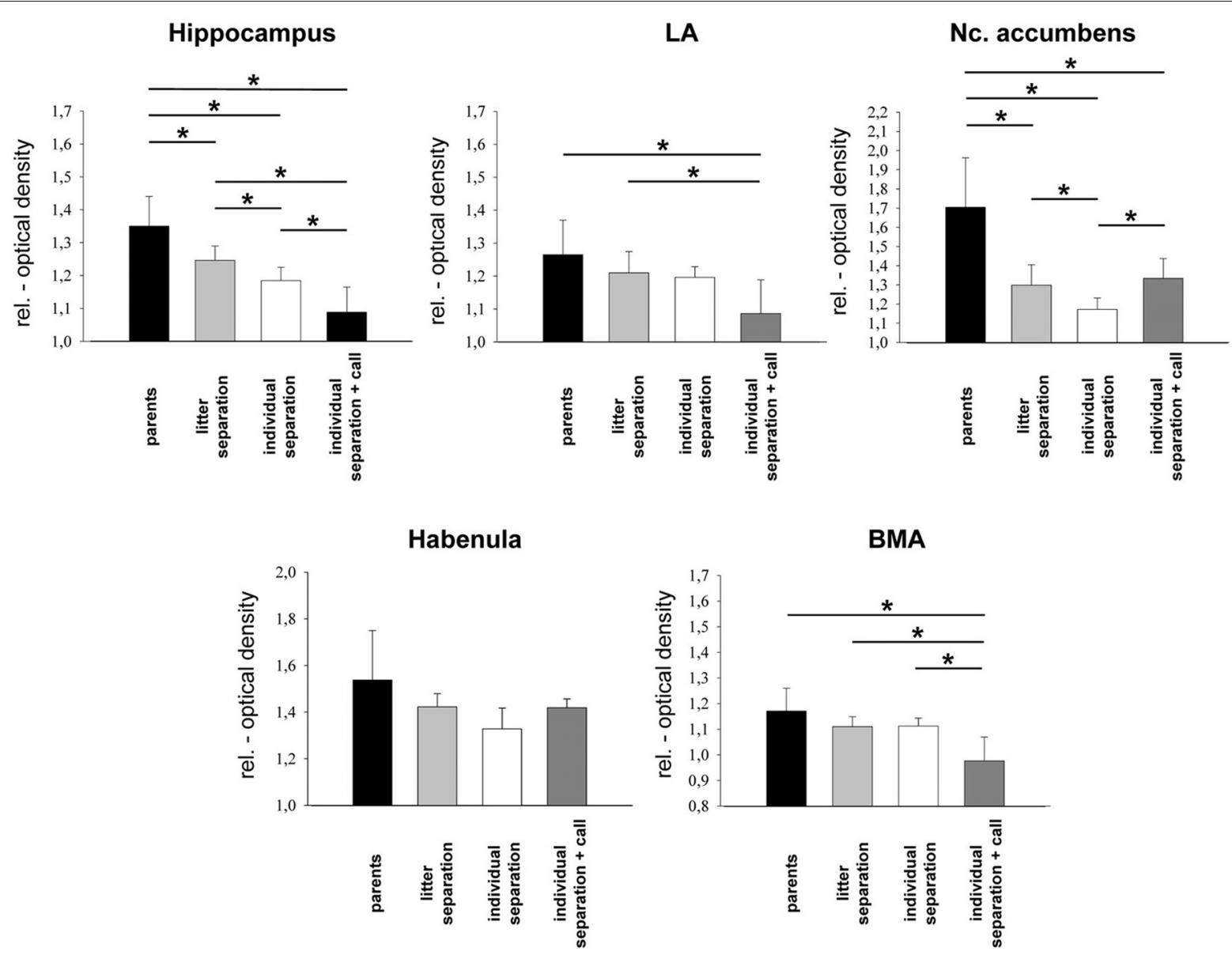

FIGURE 2 | Relative optical density of limbic brain areas in the four experimental groups. ${ }^{*} p \leq 0.05$, Mann-Whitney $U$-test. BMA, basomedial amygdala; $L A$, lateral amygdala.

\section{Sensory and subcortical areas}

In the dorsal striatum and in the somatosensory and auditory cortex of the "individual separation-call" group the presentation of maternal vocalizations during the exposure to separation stress resulted in a significant $(p \leq 0.05)$ increase of brain activity compared to the "individual separation" group (Figure 3). Brain activity in these brain areas of the "individual separation + call" group was upregulated to the levels measured in the "litter separation" group. In the PAG of the "individual separation-call" group the presentation of maternal vocalization induced a significant decrease of metabolic brain activity compared to the "individual separation" group $(p \leq 0.05)$ and completely restored metabolic activity to the levels measured in the "litter separation" and "parents" groups (Figure 3).

\section{SEPARATION STRESS ALTERS THE FUNCTIONAL COUPLING OF METABOLIC BRAIN ACTIVITY}

Calculation of the correlation coefficients (Spearman's rho, $\alpha=$ $5 \%$ ) between the relative optical densities revealed a distinct pattern of inter-regional correlations for each experimental condition (Figures 5-10). Overall, in all experimental groups we found that the number of significant positive correlations between the analyzed brain regions was higher than the number of significant negative correlations. The highest degree of inter-regional correlation was found in the animals from the parents group (Figure 5). Among the frontal cortical areas the ACd, OFC, $\mathrm{Cg}$, and $\mathrm{PrCm}$ displayed a high degree of positive correlations with the limbic areas, particularly hippocampus, BMA, and habenula and with the analyzed subcortical and sensory areas (Figures 6-8) A high number of positive correlations could also be observed among the limbic areas (Figures 8-10), with exception of the LA and also among the subcortical and sensory areas. The PAG showed positive correlations with the OFC, hippocampus, habenula, striatum, NAcc, and the thalamus (Figure 10). The prefrontal PL and IL displayed no positive correlations to the other brain areas but PL and IL were positively correlated with each other (Figures 6 and 7)

In comparison to the animals from the parents group the pattern of inter-regional correlations changed dramatically in the litter separated and individually separated animals (Figure 5). In general, the degree of correlated activity decreased in both separation groups. In the litter separation animals this effect was most prominent for the OFC (Figure 7), the BMA (Figure 9) and the analyzed subcortical and sensory areas. For the PAG the only 

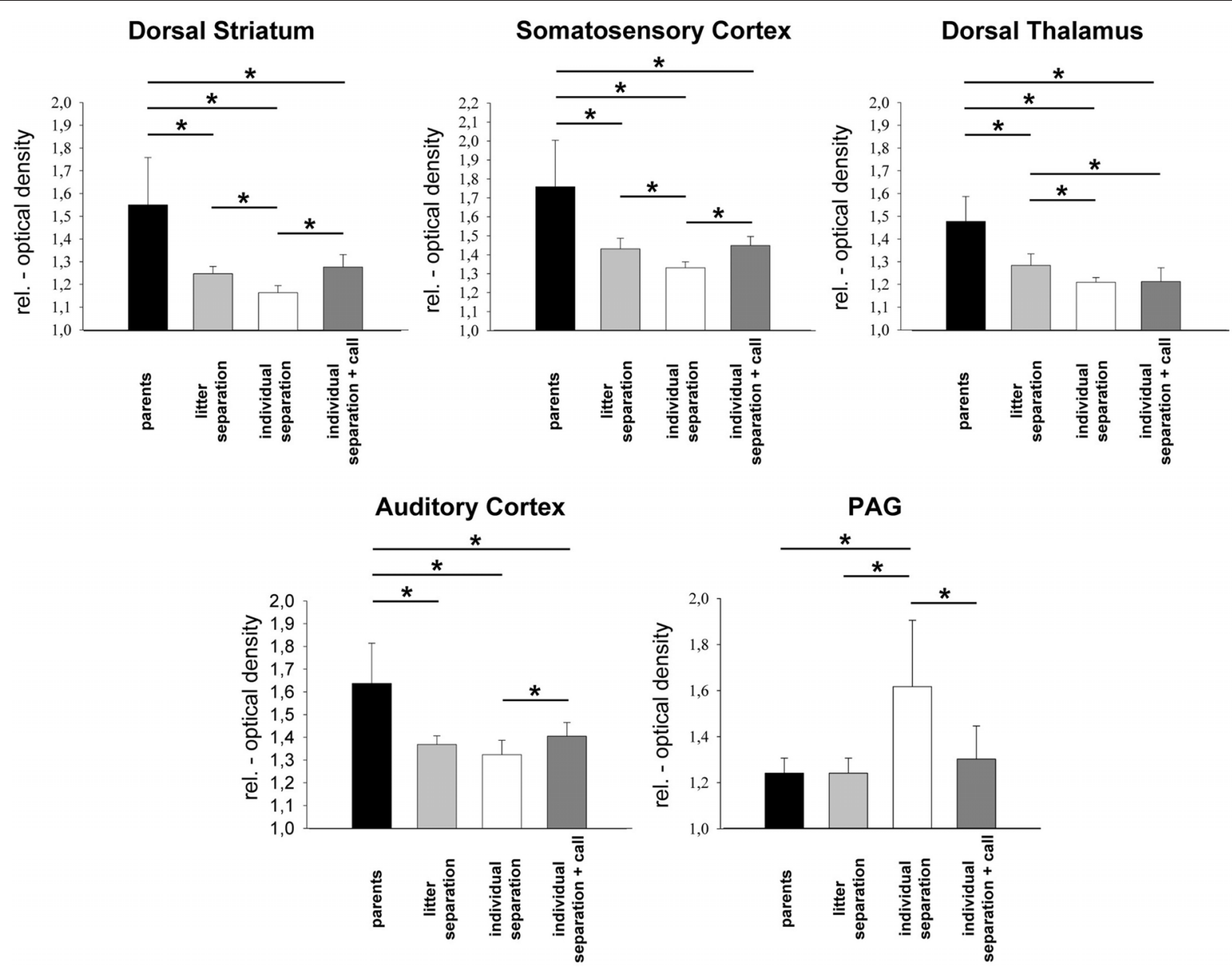

FIGURE 3 | Relative optical density of sensory and subcortical brain areas in the four experimental groups. ${ }^{*} p \leq 0.05$, Mann-Whitney $U$-test. PAG, periaqueductal gray.

positive correlations were found with the hippocampus and thalamus (Figure 10). In the individually separated animals the degree of positive inter-regional correlation was relatively low (Figure 5), however a very distinct pattern was observed since there was a strong coupling among the hippocampus, LA, and BMA, which could not be observed in the other three experimental groups (Figures 8 and 9). Also, a number of positive correlations were found for the PAG, such as positive correlations with the frontal areas ACd and PrCm, which were absent in the other experimental groups (Figure 10). Interestingly, as opposed to all other experimental conditions, a high number of negative correlations were observed in the individually separated animals, particularly between the hippocampus, LA and BMA and the frontal IL and $\mathrm{PrCm}$ as well as with the striatum and NAcc (Figures 5, 7-10). Also, the negative correlations of the PAG with the hippocampus and amygdala were only found in this experimental group (Figure 10).

A relatively low degree of inter-regional correlation was also found in the animals that were exposed to maternal vocalizations during separation (Figure 5). However, a coupling of activity was found for the frontal areas ACd, PL, IL, and OFC among each other, a pattern that was completely absent in the other experimental groups (Figures 6 and 7). Interestingly, in this group we found no correlations of the PAG with the other analyzed brain areas (Figure 10).

\section{DISCUSSION}

Although, there is clear evidence that early traumatic experiences interfere with the maturation of endocrine and behavioral function in rodents, non-human primates, and humans and thereby are risk factors for the development of later psychopathologies (Heim and Nemeroff, 2001; Sanchez et al., 2001; Stevens et al., 2009; Loman and Gunnar, 2010; Bock and Braun, 2011), there is a paucity of information about the immediate, acute effects of these adverse situations on brain activity. It was proposed that emotions are part of an evolutionarily set neural mechanism, which serves to maintain an organism's homeostasis (Damasio et al., 2000), and there is evidence that emotion-evoked neural patterns constitute multidimensional maps of the organism's internal state. 


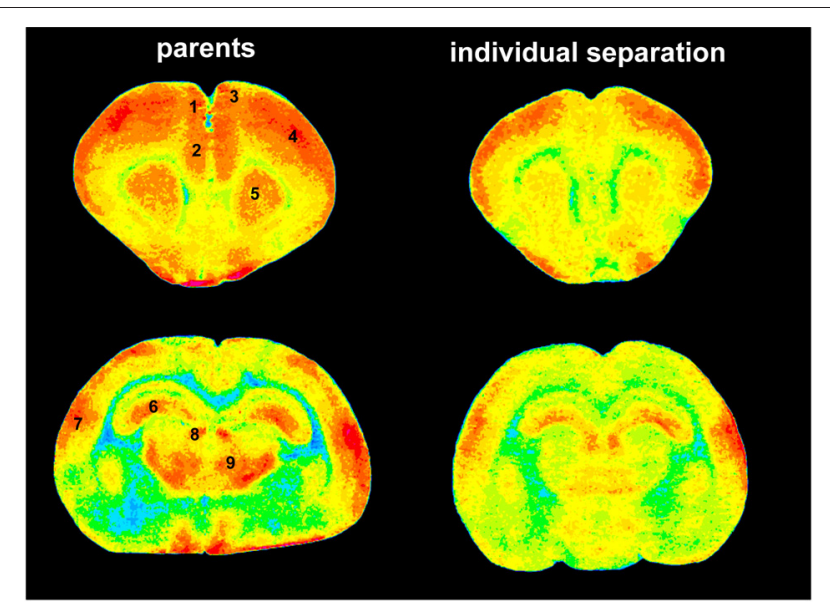

FIGURE 4 | Pseudocolor images of anterior (top) and posterior (bottom) brain slices indicating 2-FDG uptake as measurement of brain activity. Red colors indicate high levels of 2-FDG uptake (high brain activity) green and blue colors indicate low levels of 2-FDG uptake (low brain activity). Obviously, there is a dramatic decrease of brain activity in individually separated animals (right panel) compared to animals that stayed together with their parents (left panel). 1, ACd; 2, PL; 3, PrCm; 4,

somatosensory cortex; 5 , striatum; 6 , hippocampus; 7, auditory cortex; 8 , habenula; 9 , thalamus.

Thus, the aim was to identify the activity within the affective brain circuits in the infant degu brain during different separation stress conditions, and to unveil interregional functional coupling within the stress-responsive brain circuitries. Our findings reveal for the first time that stress exposure induces widespread alterations of brain activity and changes in interregional coupling in the infant brain.

\section{METHODOLOGICAL CONSIDERATIONS}

Functional imaging of emotional responses in awake, freely behaving animals is challenging since most functional imaging techniques either require the sedation of the animals (fMRI), which limits the spectrum of evocable emotional states and their detectability, or they do not provide sufficient spatial resolution (PET, SPECT) to distinguish small brain regions such as the amygdala and prefrontal cortical regions and their subdivisions. The 2-FDG autoradiography allows to image emotional brain responses while the animal is awake and actively interacts with its environment, and this technique provides sufficient spatial resolution to distinguish between subregions of the prefrontal cortex, hippocampus, the amygdala, and other areas. 2FDG is an analog of glucose, which is transported into cells by glucose transporters, phosphorylated by hexokinase and not further metabolized. The 2-FDG-phosphate accumulates intracellularly, which allows a quantitative assessment of regional glucose utilization as a measure of functional activity. Thus, the metabolic activity, which is measured, reflects both, glial and neuronal activity because neurons recruit their energy from direct glucose uptake as well as indirect glucose utilization via uptake of astrocytic lactate (Chih et al., 2001; Magistretti, 2006). One limitation of this method is that it only allows group comparisons of activity changes since it is not possible to test different time points in the same animal, and therefore the stress-induced changes cannot directly be correlated with baseline levels in the same individual.

The analysis of interregional correlations provides a sensitive tool for detecting subtle changes in functional coupling of activity in a region-specific manner (e.g., the differential correlation patterns of the two amygdala subregions in the "parent" situation). As cautionary note it should be pointed out that the large number of correlations and the small $\mathrm{N}$ per group may on one hand raise the possibility of spurious effects in some brain areas and on the other hand of insufficient N/power for detecting significant effects in other brain areas.

\section{DECREASED BRAIN ACTIVITY DURING SEPARATION STRESS}

Separation of the entire litter from the home cage and both parents for $1 \mathrm{~h}$ induced a dramatic downregulation of brain activity in a number of brain areas, and this effect was much more pronounced in pups, which were individually separated from their siblings. Decreased activity was found in all analyzed frontal and sensory cortical areas, which supports the view that, similar to humans, emotionality in animals also includes cortico-perceptive aspects (Panksepp, 2003; Wright and Panksepp, 2011). In addition, decreased activity was detected in the hippocampus and in the striatum including NAcc, and in the thalamus. As stated by Panksepp (2003) emotional processes include motor-expressive (e.g., striatum), sensory-perceptual (e.g., somatosensory, AC, thalamus), autonomic-hormonal, cognitive-attentional (prefrontal regions), and affective-feeling (e.g., cingulate, n. accumbens, PAG and amygdala) aspects. The altered activity and functional coupling (see below) observed in the stressed animals shows that many of the brain regions, which mediate these different components of emotionality are "shut down" during stress, which may be indicative of a "panic-type" emotional status. This interpretation is supported by the observation that, in contrast to all other brain areas, metabolic activity was increased in the PAG in the most severely stressed group (individual separation) and also reflected by distress vocalizations (unpublished observations). The PAG is a key component of the basic emotional systems contributing to basic emotions in the mammalian brain such as negative emotions as rage, fear and panic, but also positive emotions as lust, care and play (Panksepp, 2011). In particular, the PAG is a mediator of different defensive responses such as freezing during fear and anxiety in adult as well as in young animals (Graeff, 2004; Brandão et al., 2008; Wiedenmayer, 2009).

Acute decreases of local cerebral glucose uptake, as a measure of neuronal activity, were also described in adult mice as an acute consequence of restraint stress (Warnock and Steckler, 2011). Similar to our findings in degu pups restraint stress reduced local cerebral glucose uptake in frontal cortical areas, the thalamus, and the hippocampus. In line with our findings, a recent study using cytochrome oxidase activity as a measure of long-term changes in brain metabolic capacity after 2 weeks of exposure to repeated separation stress, reports decreased activity in the medial prefrontal cortex and NAcc in two week old mouse pups (Spivey et al., 2011). In addition, fMRI and PET studies revealed deactivation of the hippocampus, medio-OFC and anterior cingulate cortex in human subjects during exposure to a psychosocial stressor (Pruessner et al., 2008). The deactivation of the hippocampus 

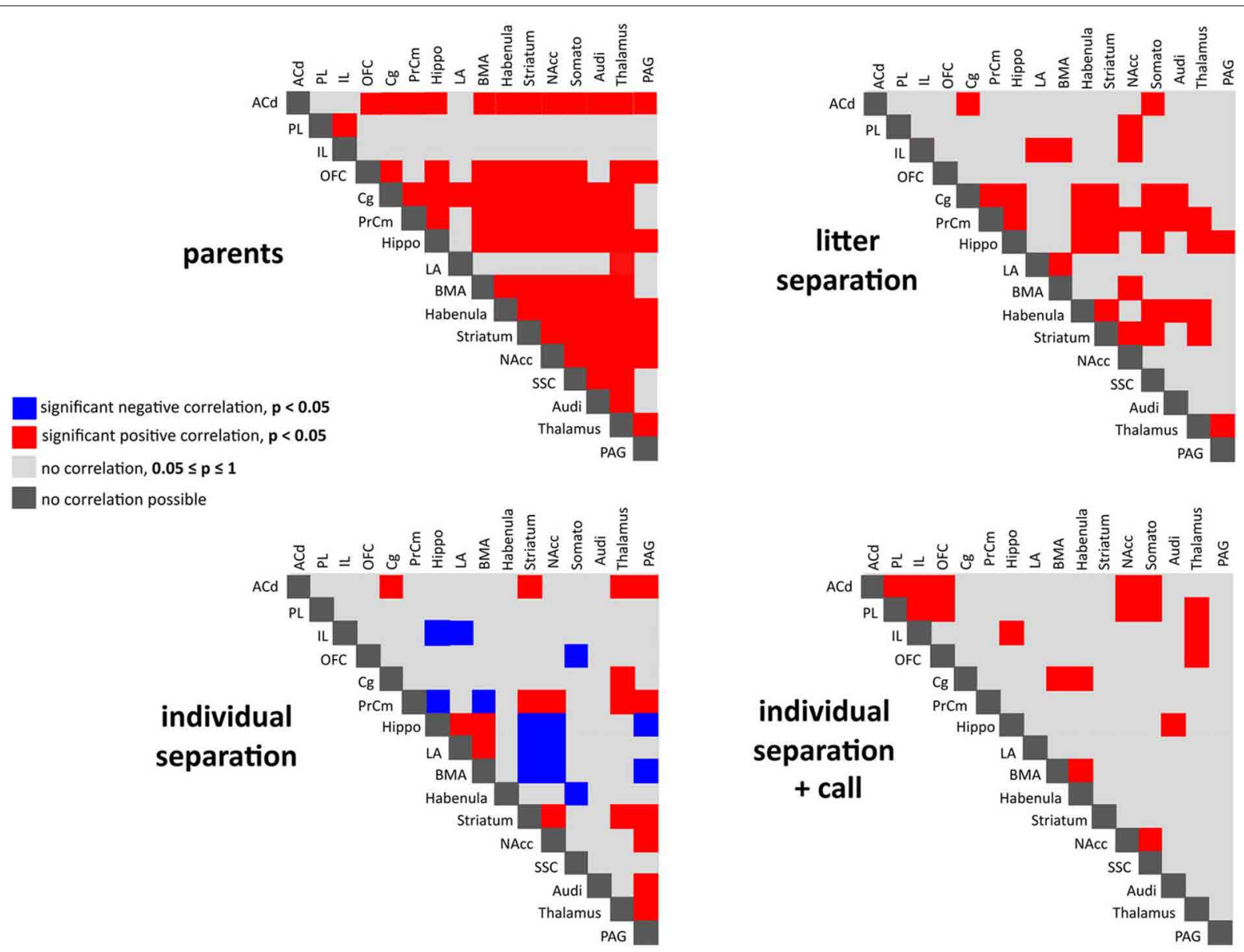

FIGURE 5 | Interregional correlations of metabolic brain activity reflecting functional coupling of the analyzed brain areas in the four experimental groups. Significant positive (red) and negative (b/ue) correlations analyzed using Spearman's rank correlation coefficient ( $p<0.05)$.

in this study was directly correlated with the release of cortisol in response to the stress task. Therefore, it is tempting to speculate that the observed deactivations in our study may be caused by high levels of stress hormones such as cortisol. This hypothesis is supported by our findings that show dramatic increases of cortisol during separation stress in degu pups (Gruss et al., 2006). There is some evidence in hippocampal cell cultures and in peripheral tissues that glucocorticoids can inhibit glucose uptake into neurons and astrocytes (Munck, 1971; Horner et al., 1990; Virgin et al., 1991).

Surprisingly, some of the major emotional brain areas, including the amygdala and the habenula did not show metabolic changes in response to separation stress. This is in line with findings from 2-DG studies using swim stress in adult rodents and a PET study in infant monkeys using separation stress, where no changes of amygdala activity could be identified (Duncan et al., 1993; Rilling et al., 2001). Since there is evidence that fear-related amygdala activity attenuates with time (LaBar et al., 1998; Phelps et al., 1998), the stress-induced changes in the amygdala may either not be detectable with the PET and 2-DG techniques due to their limited time resolution (Rilling et al., 2001), or the amygdala just does not respond under these experimental stress conditions, perhaps also due to its immaturity in the infant brain (but see results of the correlation analyzes).

The higher variability of the animals in the "family" group compared to the other groups may be due to the different social interactions of the individual pup within the family setting during the 2-FDG experiment. While the behavioral options of the stressed animals were much more limited and focused on the stressful situation resulting in a more coherent activation pattern, the behavior of the pups in the "family" group may have been more variable, e.g., some pups might have been cuddling with a parent or siblings (low activity), others might have been playing (higher activity).

\section{MOTHER'S VOICE CHANGES BRAIN ACTIVITY DURING SEPARATION}

The maternal voice is a positive emotional stimulus (Braun and Scheich, 1997; Poeggel and Braun, 1997; Braun and Poeggel, 2001), which on the behavioral level has been shown to exert an "anxiolytic" effect (Braun et al., 2003). Thus, another aim was to test the hypothesis that mother's vocalizations should "buffer," that is, ameliorate, or normalize stress-induced metabolic changes. As predicted we found that maternal vocalizations induced an increase in brain activity in the prefrontal 


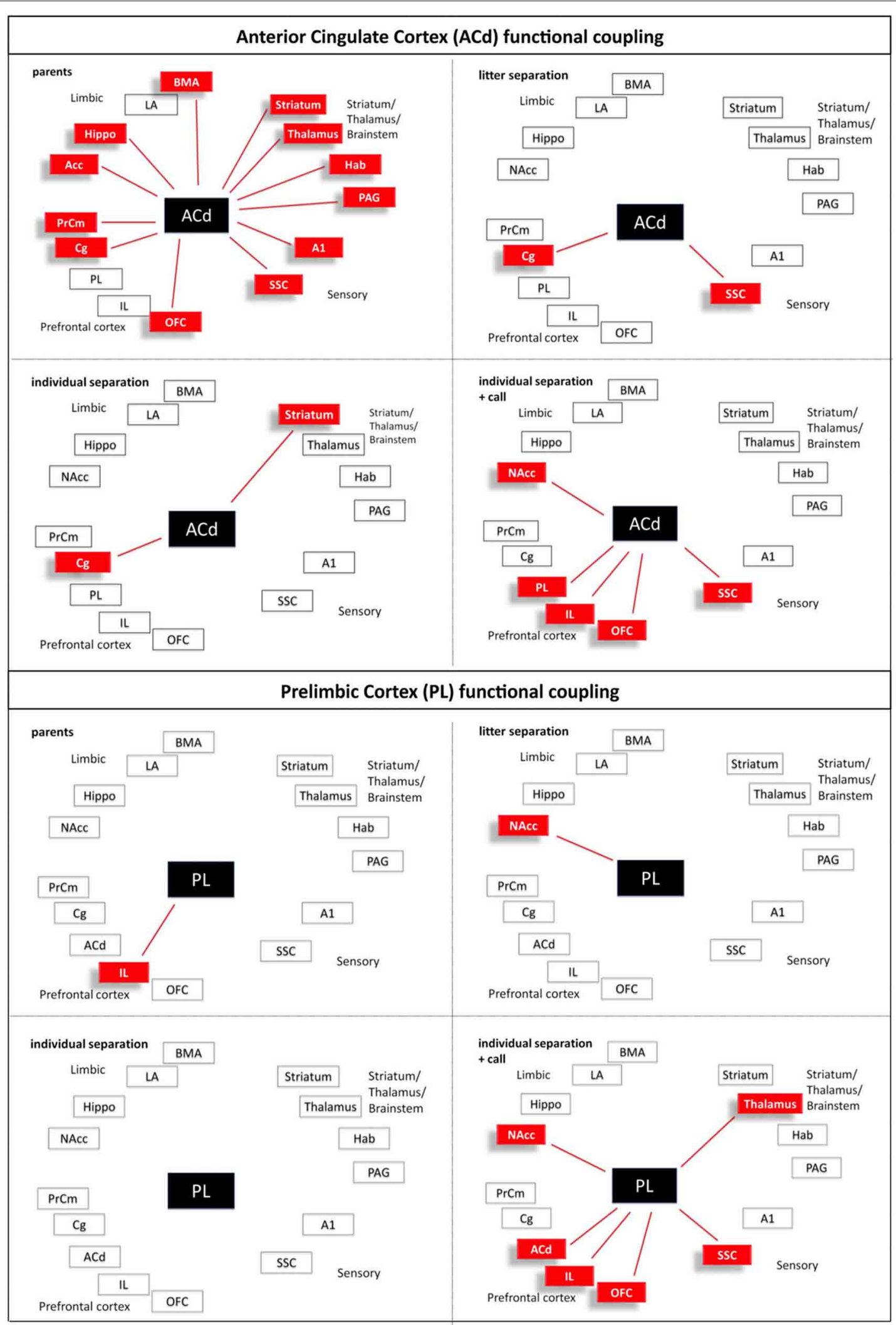

FIGURE 6 | Interregional functional coupling of the anterior cingulate (ACd) and prelimbic (PL) cortex under different stress conditions. Red color indicates positive correlations $(p<0.05)$. 


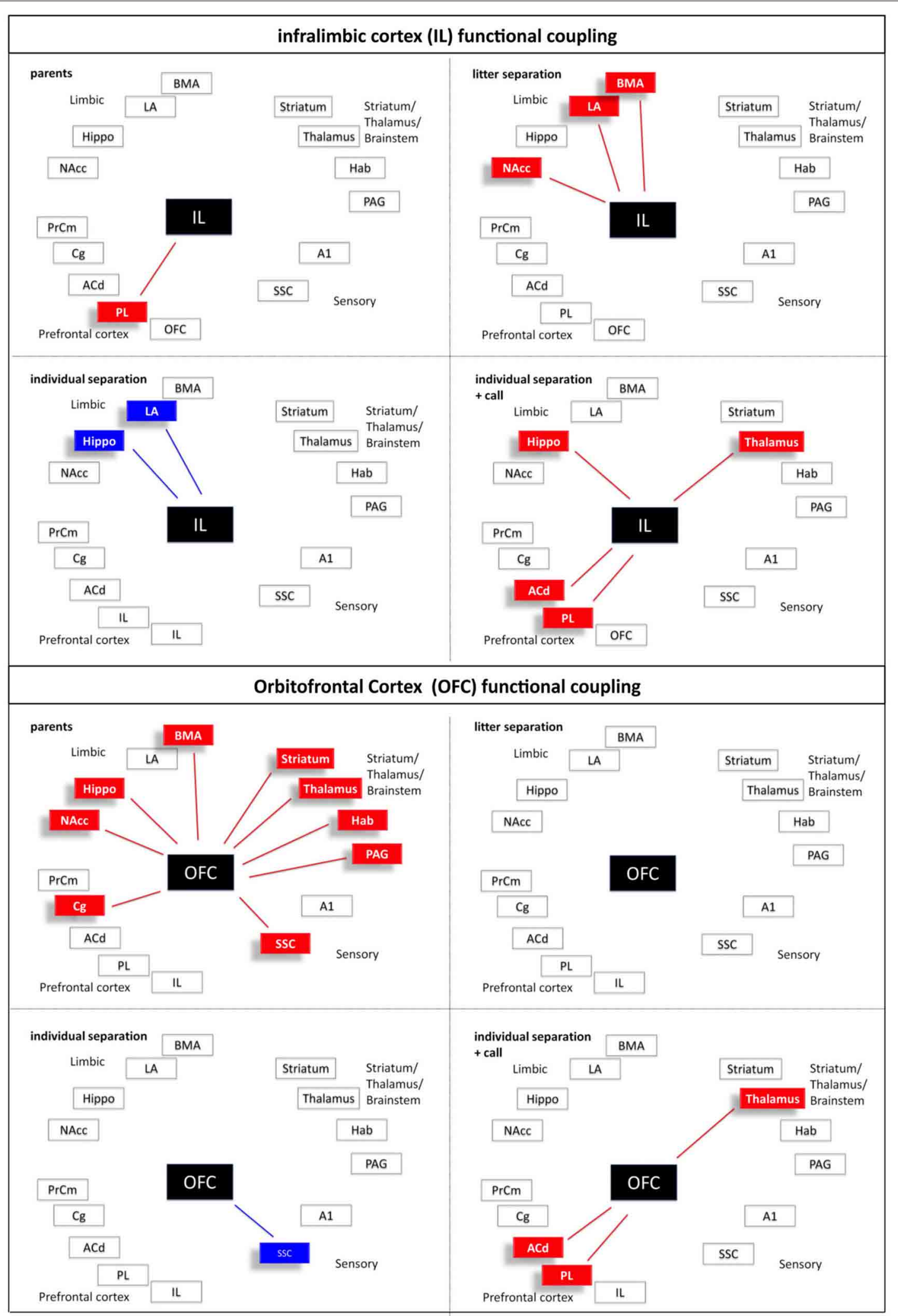

FIGURE 7 | Interregional functional coupling of the infralimbic (IL) and orbitofrontal (OFC) cortex under different stress conditions. Red color indicates positive, blue color indicates negative correlations $(p<0.05)$. 


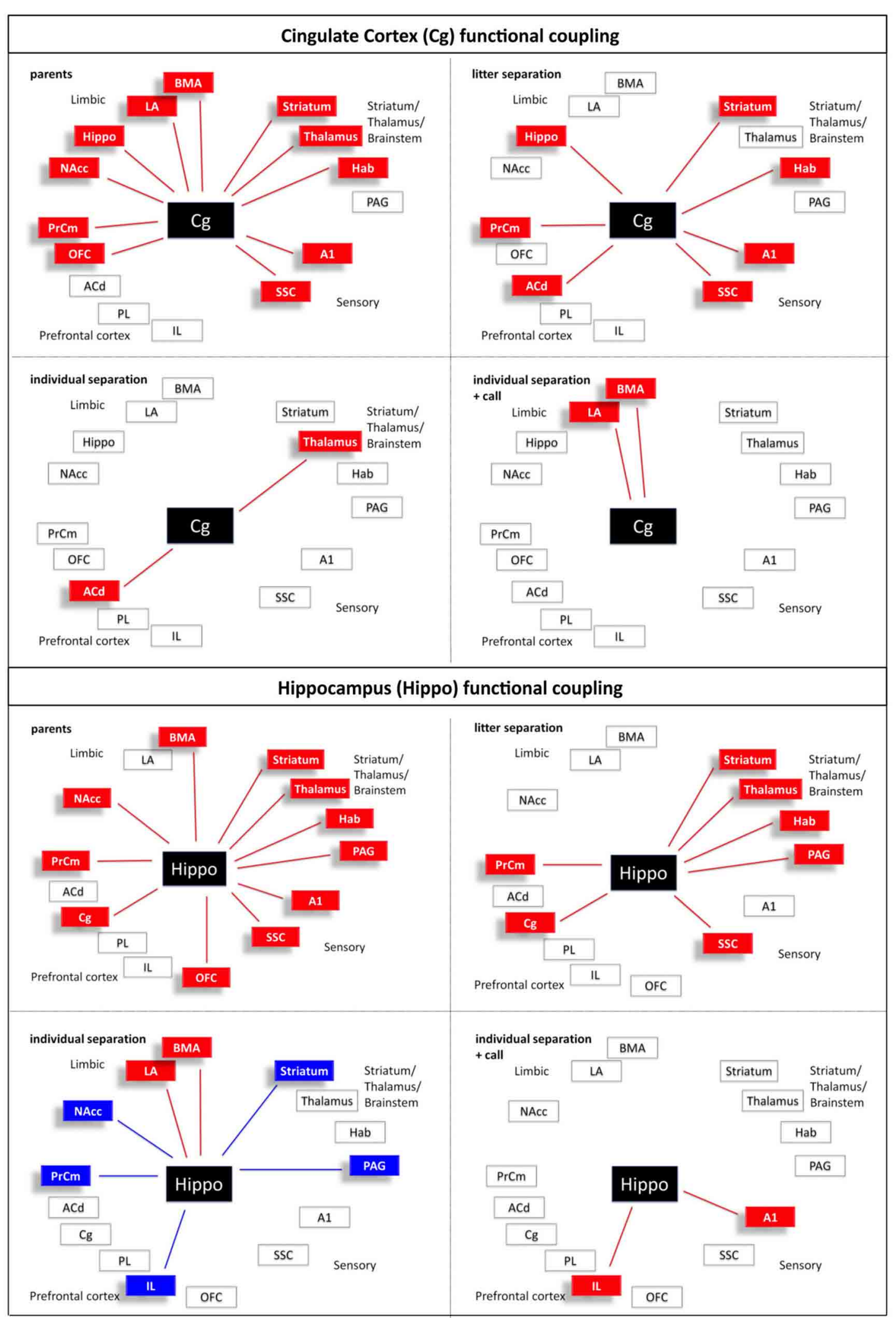

FIGURE 8 | Interregional functional coupling of the cingulate cortex $(\mathbf{C g})$ and hippocampus (Hippo) under different stress conditions. Red color indicates positive, blue color indicates negative correlations $(p<0.05)$. 


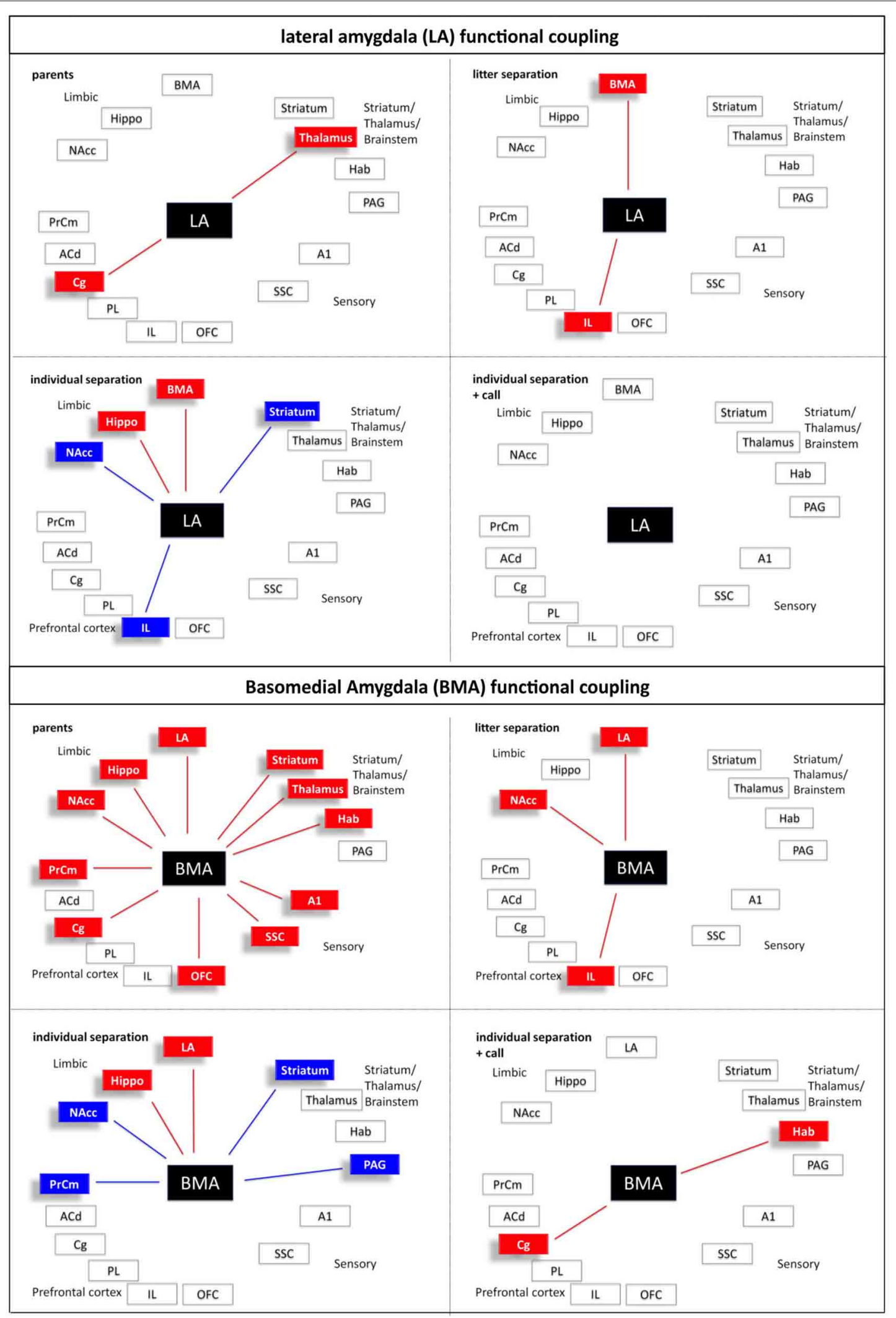

FIGURE 9 | Interregional functional coupling of the lateral (LA) and basomedial (BMA) amygdala under different stress conditions. Red color indicates positive, blue color indicates negative correlations $(p<0.05)$. 


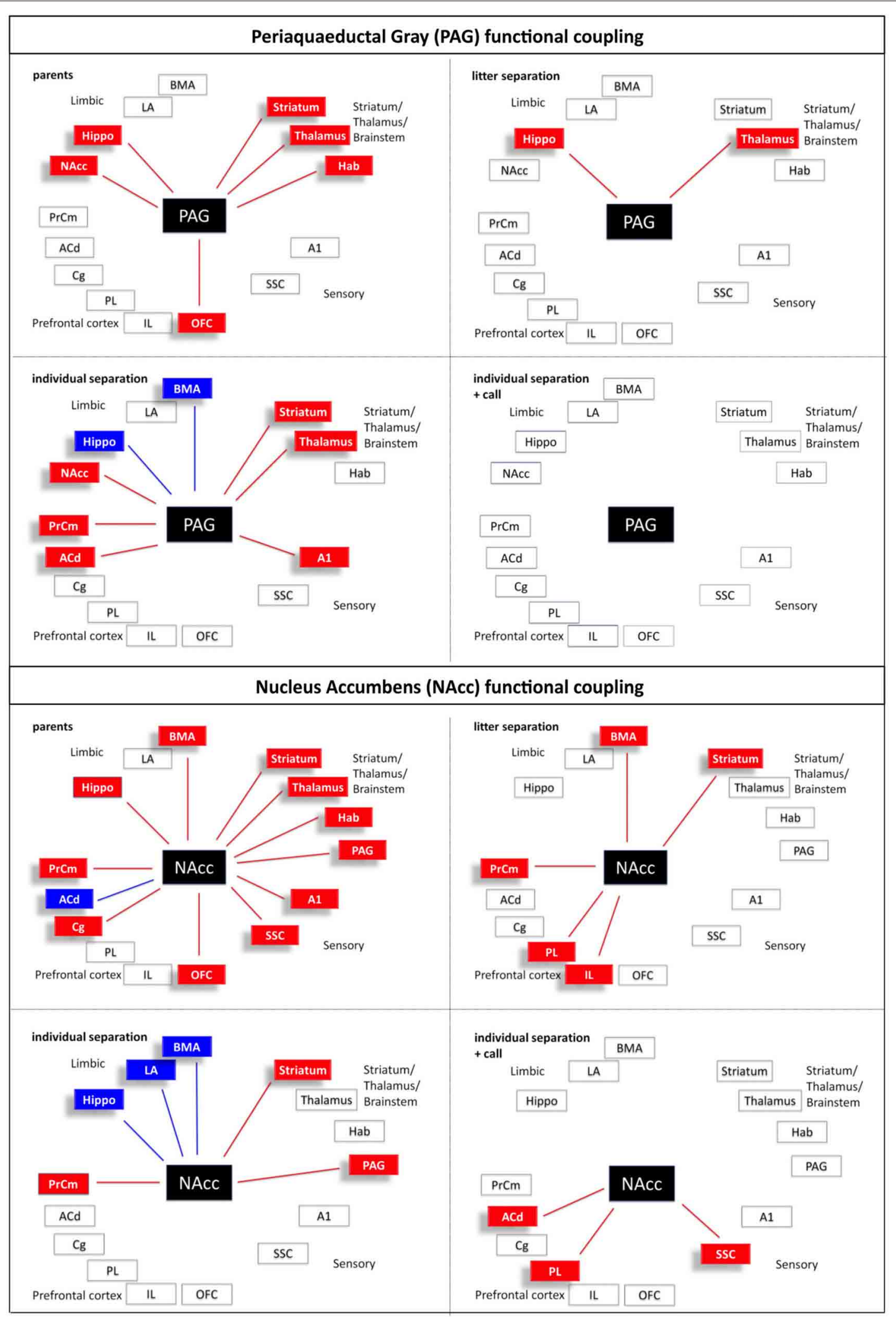

FIGURE 10 | Interregional functional coupling of the periaqueductal gray (PAG) and nucleus accumbens (NAcc) under different stress conditions. Red color indicates positive, blue color indicates negative correlations $(p<0.05)$. 
$\mathrm{Cg}$ and PrCm, the striatum and NAcc, and in the somatosensory and auditory cortex compared to the individually separated animals without acoustic stimulation. In all these brain areas brain activity was restored back to the levels of the litter separation group, but did not reach activity levels seen in the parents (control) group. In contrast, brain activity in the prefrontal PL and IL and in the limbic hippocampus and amygdala was reduced upon presentation of maternal vocalizations. The stress-induced enhanced activation of the PAG, which specifically was observed in the individual separation group and most likely reflects a panictype emotional status, was restored back to the activity levels of the litter separation and parents groups. The stress- and anxiety "buffering" effect of maternal vocalizations has also been found in other experiments where it seems to protect the infant brain from stress-induced changes. For example it has been shown in degu pups that short periods of separation stress increase the density of distinct neurotransmitter receptor subpopulations such as NMDA-, serotonin- and dopamine receptors in prefrontal ACd, PrCm, PL, and IL and hippocampus and amygdala, which were normalized by the presentation of maternal calls (Ziabreva et al., 2000, 2003a,b).

\section{ALTERED INTERREGIONAL FUNCTIONAL COUPLING DURING SEPARATION STRESS AND IN RESPONSE TO MOTHER'S VOICE}

Effective information processing, learning and also the execution of social or other complex behaviors is not restricted to the function or activity of single brain regions, but is the result of functional interactions between large-scale distributed neural systems (McIntosh and Gonzalez-Lima, 1998; Nair and GonzalezLima, 1999; Nair et al., 2001). Moreover, there is considerable evidence that sadness in humans evokes a similar arousal pattern as the separation distress vocalization circuit in rodents (Damasio et al., 2000; Panksepp, 2003, 2011). Some aspects of these "emotional" maps, such as those in the brainstem, may not be directly accessible to consciousness, as opposed to the prefrontal and cingulate cortex, regions that receive regulatory signals from brainstem and hypothalamus as well as sensory signals (Damasio et al., 2000). Our correlation analysis in stressed infant degus revealed that similarly complex changes in activation/inactivation and functional coupling patterns occur within the same brain circuits as those in the human brain. Furthermore, we show for the first time that a positive, "comforting" emotional stimulus (mother's voice), which ameliorates the status of stress and panic in the animal, recruits very different functional coupling patterns than those seen in the unstressed parent control group.

In line with our hypothesis the patterns of correlated activity dramatically changed in the different stress situations compared to the parent's situation. In the parents group a high level of positive interregional correlations was observed especially between the prefrontal OFC, $\mathrm{Cg}$, and PrCm and limbic areas. Also, the PAG showed positive correlations to OFC, hippocampus, habenula, striatum, NAcc, and thalamus. Overall, the number of positive correlations decreased in the two stressed (litter and individual separation) groups, indicating that the functional coupling within these circuits becomes increasingly disturbed. Particularly the OFC and the cingulate cortex, areas that are strongly related to executive function and decision-making, are almost completely uncoupled from the other brain regions. This uncoupling of prefrontal from limbic regions may indicate that during stress the prefrontal control of limbic structures such as the n. accumbens and the amygdala is lost. As a consequence, this may reduce the prefrontal cognitive control of fear- and anxiety-like behavior (distress vocalizations, freezing) and thereby change into a panic-like emotional state. This interpretation is supported by the finding that specifically during the most severe stress condition (individual separation) the PAG is functionally coupled to the amygdala (BMA subregion) in a negative manner, and in a positive manner with prefrontal subregions ( $\mathrm{ACd}$ and $\mathrm{PrCm}$ ) and the thalamus. This functional coupling may be mediated by direct connections of the PAG to the respective areas, since it receives afferent connections from the amygdala and medial prefrontal and cingulate cortex and sends efferent connections to parts of the thalamus (Linnman et al., 2012). Quite similar to our findings a fMRI human study provides evidence that an imminent threat elicits activity in the midbrain, including the PAG, that is paralleled by an increased coupling of the midbrain with the middorsal anterior cingulate cortex, and decreased coupling with the amygdala and hippocampus (Mobbs et al., 2009).

It is hypothesized that the amygdala is a key structure for integrating cognitive and emotional neuronal networks (Pessoa, 2008). Interestingly, our imaging study revealed that the two amygdala subnuclei display very different functional coupling patterns. Similar to the OFC and ACd the BMA, which in the parent group shows a widespread coupling with almost all other regions, almost completely decouples under stress (litter separation group). With increasing stress (individual separation) a few "reconnections" occur compared to the parent group (e.g., with the hippocampus and the LA), but also some negative correlations emerge (e.g., with striatal regions including n. accumbens, the PrCm and the PAG). This coupling is "given up" when maternal calls are presented, when the BMA activity only correlates with the habenula and the cingulate cortex, the latter of which may indicate that conscious attentional and interpretative components are recruited. Some of these coupling/decoupling effects may be mediated by hormonal mechanisms. Studies in humans revealed that corticosteroid application decreased positive as well as negative functional coupling of the amygdala to brain regions involved in the initiation and maintenance of the stress response and to those, which exert executive control (Henckens et al., 2011).

In line with the view that the presentation of maternal calls as a positive emotional stimulus can ameliorate the stress level, we expected to see in the individual separation + maternal call group a partial return to the correlation patterns seen in the litter separation or parents groups. In contrast, the presentation of maternal vocalizations induced very different interregional correlations. The perception and behavioral response to the emotional acoustic stimulus recruits the positive functional coupling among the prefrontal and cingulate regions ACd, PL, IL, and OFC, which may reflect the enhanced attention and goal-directed orientation response toward the acoustic stimulus as observed in behavioral studies (Braun et al., 2003). Additional evidence for the anxiolytic effect of the maternal calls is the complete decoupling of the PAG 
from the other brain areas. The PAG is part of emotional circuits mediating fear and panic (Panksepp, 2011). This is in line with the high degree of functional coupling of the PAG with prefrontal and limbic brain areas. Reaction to the maternal calls might reduce the feelings of fear and panic in the infant pups resulting in decoupling of the PAG from the respective systems.

It is important to note that the most severe stress situation (individual separation) is the condition where the animal most likely experiences panic, and that this is the only condition in which negative functional coupling occurred. The hippocampus, the LA, and BMA are positively coupled among each other, and under the most severe stress condition these three regions become negatively coupled to major regions of the fear circuitry (medial prefrontal cortex, n. accumbens, PAG) (Rodrigues et al., 2009) and the striatum. This stress/panic-induced negative coupling has not been previously described in human or animal studies. The negative coupling patterns might either map the functional circuitries while panic is experienced, or these maps rather reflect compensatory brain mechanisms to reduce anxiety/panic and regain emotional homeostasis.

\section{CONCLUSIONS}

A concerted interregional activity is crucial for the activitydependent maturation of cortical networks, and disturbances or deviations of these networks are related to neurodevelopmental disorders and neurological disease (Uhlhaas et al., 2009; Stam and van Straaten, 2012). Thus, deviations in functional coupling such as those occurring during separation stress, and most likely also under repeated or chronic stress conditions interfere with these developmental processes and eventually may result in dysfunctional neuronal networks and psychopathological behavioral disorders. Along this line dysfunctions of functional connectivity particularly of prefrontal and limbic regions are discussed in the context of a number of disorders in humans such as depression, obsessive-compulsive disorder, autism, schizophrenia, and Alzheimer's disease (Bassett and Bullmore, 2009; Minshew and Keller, 2010; Del Casale et al., 2011; Hulvershorn et al., 2011; Liston et al., 2011).

\section{ACKNOWLEDGMENTS}

The authors would like to thank Ute Kreher and Susann Becker for excellent technical assistance. This research was supported by grants from the Bundesministerium für Bildung und Forschung (TUR 10/I48 to Katharina Braun and 01KR1207D to Jörg Bock), the German-Israeli Foundation (G-1114-101.4/2010) and a grant from the federal state of Saxony-Anhalt and the "European Regional Development Fund" (ERDF 2007-2013), Vorhaben: Center for Behavioral Brain Sciences (CBBS).

\section{REFERENCES}

Bassett, D. S., and Bullmore, E. T. (2009). Human brain networks in health and disease. Curr. Opin. Neurol. 22, 340-347.

Bock, J., and Braun, K. (2011). The impact of perinatal stress on the functional maturation of prefrontocortical synaptic circuits: implications for the pathophysiology of ADHD? Prog. Brain Res. 189, 155-169.

Bock, J., Schnabel, R., and Braun, K. (1997). Role of the dorso-caudal neostriatum in filial imprinting of the domestic chick: a pharmacological and autoradiographical approach focused on the involvement of NMDAreceptors. Eur. J. Neurosci. 9, 1262-1272.

Bock, J., Wolf, A., and Braun, K. (1996). Influence of the N-methylD-aspartate receptor antagonist DL2-amino-5-phosphonovaleric acid on auditory filial imprinting in the domestic chick. Neurobiol. Learn. Mem. 65, 177-188.

Brandão, M. L., Zanoveli, J. M., Ruiz-Martinez, R. C., Oliveira, L. C., and Landeira-Fernandez, J. (2008). Different patterns of freezing behavior organized in the periaqueductal gray of rats: association with different types of anxiety. Behav. Brain Res. 188, $1-13$.
Braun, K., and Bock, J. (2011). The experience-dependent maturation of prefronto-limbic circuits and the origin of developmental psychopathology: implications for the pathogenesis and therapy of behavioural disorders. Dev. Med. Child Neurol. 53, 14-18.

Braun, K., Kremz, P., Wetzel, W., Wagner, T., and Poeggel, G. (2003). Influence of parental deprivation on the behavioural development in Octodon degus: modulation by maternal vocalizations. Dev. Psychobiol. 42, 237-245.

Braun, K., and Poeggel, G. (2001). Recognition of mother's voice evokes metabolic activation in the medial prefrontal cortex and lateral thalamus of Octodon degus pups. Neuroscience 103, 861-864.

Braun, S., and Scheich, H. (1997). Influence of experience on the representation of the "mothering call" in frontoparietal and auditory cortex of pups of the rodent Octodon degus: FDG mapping. J. Comp. Physiol. A 181, 697-709.

Chih, C. P., Lipton, P., and Roberts, E. L. Jr. (2001). Do active cerebral neurons really use lactate rather than glucose? Trends Neurosci. 24, 573-578.

Colonnello, V., Iacobucci, P., Fuchs, T., Newberry, R. C., and Panksepp, J. (2011). Octodon degus. A useful animal model for socialaffective neuroscience research: basic description of separation distress, social attachments and play. Neurosci. Biobehav. Rev. 35, 1854-1863.

Damasio, A. R., Grabowski, T. J., Bechara, A., Damasio, H., Ponto, L. L., Parvizi, J., and Hichwa, R. D. (2000). Subcortical and cortical brain activity during the feeling of self-generated emotions. Nat. Neurosci. 3, 1049-1056.

Del Casale, A., Kotzalidis, G. D., Rapinesi, C., Serata, D., Ambrosi, E., Simonetti, A., Pompili, M., Ferracuti, S., Tatarelli, R., and Girardi P. (2011). Functional neuroimaging in obsessive-compulsive disorder. Neuropsychobiology 64, 61-85.

Duncan, G. E., Johnson, K. B., and Breese, G. R. (1993). Topographic patterns of brain activity in response to swim stress: assessment by 2 deoxyglucose uptake and expression of Fos-like immunoreactivity. J. Neurosci. 13, 3932-3943.

Fuchs, E., Flugge, G., and Czeh, B. (2006). Remodeling of neuronal networks by stress. Front. Biosci. 11, 2746-2758.

Fuchs, T., Lacobucci, P., MacKinnon, K. M., and Panksepp, J. (2010). Infantmother recognition in a social rodent (Octodon degus). J. Comp. Psychol. 124, 166-175.
Gonzalez-Lima, F. (1992). "Brain imaging of auditory learning functions in rats: studies with fluorodeoxyglucose autoradiography and cytochrome oxidase histochemistry", in Advances in Metabolic Mapping Techniques for Brain Imaging of Behavioral and Learning Functions, eds F. Gonzalez-Lima, F. T. Finkenstädt, and H. Scheich (Kluwer Academic Publishers), 39-109.

Gonzalez-Lima, F., and Scheich, H. (1986). Neural substrates for tone-conditioned bradycardia demonstrated with 2-deoxyglucose. II. Auditory cortex plasticity. Behav. Brain Res. 20, 281-293.

Graeff, F. G. (2004). Serotonin, the periaqueductal gray and panic. Neurosci. Biobehav. Rev. 28, 239-259.

Gruss, M., Westphal, S., Luley, C., and Braun, K. (2006). Endocrine and behavioural plasticity in response to juvenile stress in the semi-precocial rodent Octodon degus. Psychoneuroendocrinology 31, 361-372.

Guterman, A., and Richter-Levin, G. (2006). Neuromodulators of LTP and NCAMs in the amygdala and hippocampus in response to stress. EXS 98, 137-148.

Heim, C., and Nemeroff, C. B. (2001). The role of childhood trauma in the neurobiology of mood and 
anxiety disorders: preclinical and clinical studies. Biol. Psychiatry 49, 1023-1039.

Heim, C., Shugart, M., Craighead, W. E., and Nemeroff, C. B. (2010). Neurobiological and psychiatric consequences of child abuse and neglect. Dev. Psychobiol. 52, 671-690.

Henckens, J. A. G., van Wingen, G. A., Joels, M., and Fernandez, G. (2011). Corticosteroid induced decoupling of the amygdala in men. Cereb. Cortex. [Epub ahead of print].

Horner, H. C., Packan, D. R., and Sapolsky, R. M. (1990). Glucocorticoids inhibit glucose transport in cultured hippocampal neurons and glia. Neuroendocrinology 52, 57-64.

Hulvershorn, L. A., Cullen, K., and Anand, A. (2011). Toward dysfunctional connectivity: a review of neuroimaging findings in pediatric major depressive disorder. Brain Imaging Behav. 5, 307-328.

Korosi, A., and Baram, T. Z. (2009). The pathways from mother's love to baby's future. Front. Behav. Neurosci. 3:27. doi: 10.3389/neuro.08.027.2009

LaBar, K. S., Gatenby, J. C., Gore, J. C., LeDoux, J. E., and Phelps, E. A. (1998). Human amygdala activation during conditioned fear acquisition and extinction: a mixed-trial fMRI study. Neuron 20, 937-945.

Linnman, C., Moulton, E. A., Barmettler, G., Becerra, L., and Borsook, D. (2012). Neuroimaging of the periaqueductal gray: state of the field. Neuroimage 60, 505-522.

Liston, C., Cohen, M. M., Teslovich, T., Levenson, D., and Casey, B. J. (2011). Atypical prefrontal connectivity in attentiondeficit/hyperactivity disorder: pathway to disease or pathological end point? Biol. Psychiatry 69, 1168-1177.

Loman, M. M., and Gunnar, M. R. (2010). Early experience and the development of stress reactivity and regulation in children. Neurosci. Biobehav. Rev. 34, 867-876.

Magistretti, P. J. (2006). Neuron-glia metabolic coupling and plasticity. J. Exp. Biol. 209, 2304-2311.

McEwen, B. S. (2010). Stress, sex, and neural adaptation to a changing environment: mechanisms of neuronal remodeling. Ann. N.Y. Acad. Sci. 1204 (Suppl.), E38-E59.

McIntosh, A. R., and Gonzalez-Lima, F. (1998). Large-scale functional connectivity in associative learning: interrelations of the rat auditory, visual, and limbic systems. J. Neurophysiol. 80, 3148-3162.

Minshew, N. J., and Keller, T. A. (2010). The nature of brain dysfunction in autism: functional brain imaging studies. Curr. Opin. Neurol. 23, 124-130.

Mobbs, D., Marchant, J. L., Hassabis, D., Seymour, B., Tan, G., Gray, M., Petrovic, P., Dolan, R. J., and Frith, C. D. (2009). From threat to fear: the neural organization of defensive fear systems in humans. J. Neurosci. 29, 12236-12243.

Moriceau, S., and Sullivan, R. M. (2006). Maternal presence serves as a switch between learning fear and attraction in infancy. Nat. Neurosci. 9, 1004-1006.

Munck, A. (1971). Glucocorticoid inhibition of glucose uptake by peripheral tissues: old and new evidence, molecular mechanisms, and physiological significance. Perspect. Biol. Med. 14, 265-269.

Nair, H. P., Berndt, J. D., Barrett, D., and Gonzalez-Lima, F. (2001). Maturation of extinction behavior in infant rats: large-scale regional interactions with medial prefrontal cortex, orbitofrontal cortex, and anterior cingulate cortex. J. Neurosci. 21, 4400-4407.

Nair, H. P., and Gonzalez-Lima, F. (1999). Extinction of behavior in infant rats: development of functional coupling between septal, hippocampal, and ventral tegmental regions. J. Neurosci. 19, 8646-8655.

Neumann, I. D., Wegener, G., Homberg, J. R., Cohen, H., Slattery, D. A., Zohar, J., Olivier, J. D., and Mathé, A. A. (2010). Animal models of depression and anxiety: what do they tell us about human condition? Prog. Neuropsychopharmacol. Biol. Psychiatry 35, 1357-1375.

Panksepp, J. (2003). At the interface of the affective, behavioral, and cognitive neurosciences: decoding the emotional feelings of the brain. Brain Cogn. 52, 4-14.

Panksepp, J. (2011). The basic emotional circuits of mammalian brains: do animals have affective lives? Neurosci. Biobehav. Rev. 35, 1791-1804.

Paxinos, G., and Watson, W. (1998). The Rat Brain in Stereotaxic Coordinates, 4th Edn. San Diego, CA: Academic Press.

Pechtel, P., and Pizzagalli, D. A. (2010). Effects of early life stress on cognitive and affective function: an integrated review of human literature. Psychopharmacology (Berl.) 214, 55-70.
Pessoa, L. (2008). On the relationship between emotion and cognition. Nat. Rev. Neurosci. 9, 148-158.

Phelps, E. A., O’Connor, K. J., Gatenby, J. C., Gore, J. C., Grillon, C., and Davis, M. (1998). Activation of the human amygdala by a cognitive representation of fear. Neuroimage S8, 9 .

Poeggel, G., and Braun, K. (1997). Early auditory filial learning in degus (Octodon degus): behavioral and autoradiographic studies. Brain Res. 743, 162-170.

Pruessner, J. C., Dedovic, K., KhaliliMahani, N., Engert, V., Pruessner M., Buss, C., Renwick, R., Dagher, A., Meaney, M. J., and Lupien, S. (2008). Deactivation of the limbic system during acute psychosocial stress: evidence from positron emission tomography and functional magnetic resonance imaging studies. Biol. Psychiatry 63, 234-240.

Pryce, C. R., Aubert, Y., Maier, C., Pearce, P. C., and Fuchs, E. (2011). The developmental impact of prenatal stress, prenatal dexamethasone and postnatal social stress on physiology, behaviour and neuroanatomy of primate offspring: studies in rhesus macaque and common marmoset. Psychopharmacology (Berl.) 214, 33-53.

Riedel, A., Gruss, M., Bock, J., and Braun, K. (2010). Impaired active avoidance learning in infant rats appears to be related to insufficient metabolic recruitment of the lateral septum. Neurobiol. Learn. Mem. 93, 275-282.

Rilling, J. K., Winslow, J. T., O’Brien, D., Gutman, D. A., Hoffman, J. M., and Kilts, C. D. (2001). Neural correlates of maternal separation in rhesus monkeys. Biol. Psychiatry 49, 146-157.

Rodrigues, S. M., LeDoux, K. L., and Sapolsky, R. M. (2009). The influence of stress hormones on fear circuitry. Annu. Rev. Neurosci. 32, 289-313.

Sanchez, M. M., Ladd, C. O., and Plotsky, P. M. (2001). Early adverse experience as a developmental risk factor for later psychopathology: evidence from rodent and primate models. Dev. Psychopathol. 13, 419-450.

Sandi, C. (2004). Stress, cognitive impairment and cell adhesion molecules. Nat. Rev. Neurosci. 5, 917-930.

Schmidt, M. V., Wang, X. D., and Meijer, O. C. (2011). Early life stress paradigms in rodents: potential animal models of depression?
Psychopharmacology (Berl.) 214, 131-140.

Sokoloff, L., Reivich, M., Kennedy, C., Des Rosiers, M. H., Patlak, C. S., Pettigrew, K. D., Sakurada, O., and Shinohara, M. (1977). The [14C]deoxyglucose method for the measurement of local cerebral glucose utilization: theory, procedure, and normal values in the conscious and anesthetized albino rat. J. Neurochem. 28, 897-916.

Spivey, J. M., Padilla, E., Shumake, J. D., and Gonzalez-Lima, F. (2011). Effects of maternal separation, early handling, and gonadal sex on regional metabolic capacity of the preweanling rat brain. Brain Res. 1367, 198-206.

Stam, C. J., and van Straaten, E. C. (2012). The organization of physiological brain networks. Clin. Neurophysiol. Feb 20. [Epub ahead of print].

Stevens, H. E., Leckman, J. F., Coplan, J. D., and Suomi, S. J. (2009). Risk and resilience: early manipulation of macaque social experience and persistent behavioral and neurophysiological outcomes. J. Am. Acad. Child Adolesc. Psychiatry 48, 114-127.

Uhlhaas, P. J., Roux, F., Rodriguez, E., Rotarska-Jagiela, A., and Singer, W. (2009). Neural synchrony and the development of cortical networks. Trends Cogn. Sci. 14, 72-80.

Virgin, C. E. Jr., Ha, T. P., Packan, D. R., Tombaugh, G. C., Yang, S. H., Horner, H. C., and Sapolsky, R. M. (1991). Glucocorticoids inhibit glucose transport and glutamate uptake in hippocampal astrocytes: implications for glucocorticoid neurotoxicity. J. Neurochem. 57, 1422-1428.

Wallhäusser, E., and Scheich, H. (1987). Auditory imprinting leads to differential 2-deoxyglucose uptake and dendritic spine loss in the chick rostral forebrain. Brain Res. 428, 29-44.

Warnock, G. I., and Steckler, T. (2011). Stress-induced decreases in local cerebral glucose utilization in specific regions of the mouse brain. BMC Res. Notes 4, 96.

Wiedenmayer, C. P. (2009). Plasticity of defensive behavior and fear in early development. Neurosci. Biobehav. Rev. 33, 432-441.

Wright, J. S., and Panksepp, J. (2011). Toward affective circuit-based preclinical models of depression: sensitizing dorsal PAG arousal leads to sustained suppression of positive affect in rats. Neurosci. Biobehav. Rev. 35, 1902-1915. 
Ziabreva, I., Poeggel, G., Schnabel, R., and Braun, K. (2003a). Separationinduced receptor changes in the hippocampus and amygdala of Octodon degus: influence of maternal vocalizations. J. Neurosci. 23, 5329-5336.

Ziabreva, I., Schnabel, R., and Braun, K. (2000). Parental deprivation induces N-methylD-aspartate-receptor upregulation in limbic brain areas of Octodon degus: protective role of the maternal call. Neural. Plast. 7, 233-244.

Ziabreva, I., Schnabel, R., Poeggel, G., and Braun, K. (2003b). Mother's voice "buffers" separation-induced receptor changes in the prefrontal cortex of octodon degus. Neuroscience 119, 433-441.

Conflict of Interest Statement: The authors declare that the research was conducted in the absence of any commercial or financial relationships that could be construed as a potential conflict of interest.

Received: 02 March 2012; paper pending published: 21 March 2012; accepted: 14 April 2012; published online: 10 May 2012

Citation: Bock J, Riedel A and Braun K (2012) Differential changes of metabolic brain activity and interregional functional coupling in prefronto-limbic pathways during different stress conditions: functional imaging in freely behaving rodent pups. Front. Cell. Neurosci. 6:19. doi: 10.3389/fncel. 2012.00019

Copyright (c) 2012 Bock, Riedel and Braun. This is an open-access article distributed under the terms of the Creative Commons Attribution Non Commercial License, which permits non-commercial use, distribution, and reproduction in other forums, provided the original authors and source are credited. 